[10] Sommer. Proc. Worls'd Dairy Congress, 1923, 974-985.

[11] Rogers loc. eit.

[12] Ritter cit. ErLandsen. Dairy Industries, 3, 1938, 302-304.

[13] W. L. Davies cit. Jensen. Le Lait, 18, $1938,760$.

[14] Voir mon travail précédent.

[15] Weinziri. Journal Med. Res., 39, $1919,349$.

[16] Cooksey eit. Hunziker, Condensed Milk, $4^{\mathrm{e}}$ éd, 352.

[17] Laffrogue et Andrieu. Le Lait, 16, 1936, 1108.

[18] GoRINI. Latte Latticini, 12, 1936, 30-35.

[19] Rodelia cit. WeINBERG. Les microbes anaérobies, 1937, 594.

\title{
SUR LA CONGÉLATION DU LAIT
}

\author{
par \\ Albert FOURNIER \\ Ancien próparateur titulaire à la Sorbonne.
}

Dans un rapport et une communication présentés, le premier, au "Congrès international de la Laiterie " (1), la seconde, à la "Société de Chimie biologique " (2), j'ai apporté une contribution à l'étude des effets, sur le lait, des variations de température. En particulier, j'ai montré que la congélation du lait maintenue à $-15^{\circ}$ centigrades pendant 24 heures, n'avait aucune influence sur son acidité lactique primitive ni sur la valeur de ses diastases. A priori, aueune surprise ne pouvait en résulter. En effet, à cette température, tous les constituants du lait sont à l'état solide. L'influence chimique réciproque des corps à l'état solide mélangés est généralement nulle, surtout quand il s'agit de produits animaux et végétaux ou de leurs dérivés. Leurs réactions mutuelles, au surplus, exigent la présence de l'eau, et de l'eau à l'état liquide ou tout au moins à l'état de vapeur. Or, à $-15^{\circ}$, l'eau est solide. Elle est; de plus, cristallisée, et l'on sait que dans un mélange liquide, la cristallisation d'un de ses composants entraîne une séparation qui tend vers son isolement. C'est même un procédé de concentration du lait qui n'entraîne plus de perte d'aucun de ses prineipes comme il arrive par l'emploi de l'évaporation, même dans le vide à basse température. Il suffit de séparer les cristaux de glace, méthode que j'ai généralisée à la concentration des extraits des différents organes (3). Dans les cas envisagés ici, il est vrai, les mélanges et les combinaisons sont préexistants à la congélation, laquelle entraîne une modification de leur équilibre physique sinon chimique, équilibre que la décongélation peut ne pas rétablir dans son intégrité primitive.

(1) Berlin, août 1937 ,

(2) Séance du 4 Janvier 1938 ,

(3) Brevet du 22 novembre $1929, n^{\circ} 285.377$. 
On sait que la viande décongelée s'altère plus vite que la viande fraîche, et cela sans doute en raison de la destruction de son architecture cellulaire, sous l'influence probable, en particulier, de la dilatation glaciaire à basse température. Cependant il est des animaux et des plantes qui résistent à des froids rigoureux. Mais leur facteur vital est une protection inconnue des produits dépourvus d'énergie vitale, comme la viande même fraîche et le lait excrété, même récemment, de la mamelle.

Ces produits morts, recueillis aseptiquement, paraissent néanmoins se conserver indéfiniment sans modifications progressivement destructives, même à des températures où leur altération deviendrait rapidement putride; à plus forte raison en est-il ainsi quand leurs constituants et surtout leur eau de constitution sont à une température où ils se maintiennent à l'état solide.

Le lait, quoique n'étant pas, à vrai dire, d'une architecture celluláre comparable à celle de la plupart des tissus de l'économie, lors de sa décongélation, reprend-il sa composition bio-physique primitive? Dans le cas contraire, a-t-il perdu une partie de ses propriétés alimentaires et hygiéniques?

Sous l'influence du gel, des auteurs américains disent avoir constaté des modifications physiques du lait (1), et, par son dégel, des altérations diastasiques et mierobiennes allant jusqu'à la protéolyse peptonisante et acido-aminée (2). Ces auteurs conseillent de faire bouillir le lait immédiatement après son dégel avant d'en nourrir les jeunes enfants de santé délicate, sous peine de voir apparaître chez eux des troubles morbides assez graves.

On comprend très bien que des phénomènes suspendus pendant la période de gel puissent renaître au dégel; surtout s'il s'agit de lait primitivement impur. Il faudrait comparer deux échantillons d'un même lait : étudier les transformations d'un premier échantillon non soumis au gel et les comparer aux transformations d'un lait congelé pendant un certain temps. S'il y avait identité de transformations, le gel n'aurait fait qu'en suspendre les effets. Mes premières expériences, communiquées à Berlin et à la Société de Chimie biologique (voir plus haut), ont eu pour but une telle étude comparative. Dans les cas particuliers examinés par elles, j'ai pu constater la parfaite conservation du lait pendant toute la période de gel.

Mes études présentées ici, qui remontent au mois de mai 1939 et au mois de janvier 1940 , ont entièrement confirmé les résultats des premières.

Mes observations ont porté sur un volume constant de $5 \mathrm{em}^{3}$,

(1) Pb. Hennemann, Milk, Philadelphia, WB Sanders Company, 1920.

(2) Pennington, Hepburn, Witner. Stafford et Bureli, Journal Biol Chem., 16, 1913,331$)$. 
volume réduit afin de permettre une emprise plus immédiate des variations thermiques et la comparaison des résultats dans un temps minimum (1). Les acidités ont été évaluées avec $\mathrm{NaOH}, \mathrm{N} / 10$, au moyen de burettes donnant le $1 / 20^{e}$ de centimètre cube chacun d'eux représentant une goutte de $\mathrm{NaOH}, \mathrm{N} / 10$. Dans la congélation du lait, l'eau ayant une grande importance, l'étude de la dilution a été faite en effectuant des mélanges Lait + Eau $=5 \mathrm{~cm}^{3}$, où le volume de lait varie de 5 à 0 par fraction de $1 \mathrm{~cm}^{3}$, tandis qu'inversement celui de l'eau varie de 0 à 5 .

De même, les ferments lactiques jouant un rôle considérable dans les altérations primitives du lait, j'ai artificiellement enrichi le lait d'une culture active de ferments lactiques purs et sélectionnés, en faisant varier les proportions de leur mélange avec le lait dans les mêmes rapports qu'avec l'eau et le lait, sous le même volume de $5 \mathrm{~cm}^{3}$. Les tableaux ci-joints fournissent les variations de l'acidogénie. AT représente l'acidité totale de chaque mélange directement évaluée au moyen de la phtaléine du phénol; AP, la somme des acidités partielles de chacune des fractions du mélange ; I, la différence AT-AP, ou indice d'acidification, mesurant l'acidogénie dont chaque mélange a été le siège.

Les résultats numériques obtenus sur $5 \mathrm{~cm}^{3}$ des mélanges ont été rapportés par le calcul à $100 \mathrm{~cm}^{3}$, en sorte que si v et v' représentent les volumes partiels des mélanges, on aura :

$$
v+v^{\prime}=100
$$

AP sera obtenu par la formule :

$$
\mathrm{nv}+\mathrm{n}^{\prime} \mathrm{v}^{\prime}=\mathrm{AP}
$$

où n et n' représentent les coefficients fractionnaires des volumes partiels assurant le volume total de 100 .

\section{Première série d'expériences}

Dans le tableau I, les tubes 34 à 39 permettent de considérer l'acidogénie comme nulle. Aux approximations expérimentales près, on voit en effet que I est sensiblement nul.

Dans le tableau II, après 24 heures de séjour à la température ordinaire, les tubes de 11 à 16 fournissent un AT progressivement

(1) On a utilisé des tubes à essai d'un diamètre choisi ayant tous à peu près la même capacité. La vitesse de congélation, en effet, peut avoir une certaine importance sur l'équilibre final. Elle dépend du vase, de sa forme, de sa capacité; et comme elle est dirigée de dehors en dedans, une agitation constante serait désirable. On pourrait encore tendre à zéro le volume du lait à congeler, soit en le recevant en gouttes dans l'appareil congélateur, soit en réduisant son volume au maximum, en surface (ainsi qu'il est fait pour l'obtenir sec à une température supérieure à zéro) ou en ligne aussi étroite que possible. L'emprise rapide du froid a d'ailleurs moins d'importance quand sa durée d'application augmente. 
TABLEAU

DOSAGE DE L'AGIDITE A LA REGEPTION

(Température moyenne du laboratoire : 15 à $20^{\circ} \mathrm{C}$.)

\begin{tabular}{|c|c|c|c|c|c|c|c|}
\hline & Tubes & $\begin{array}{c}\mathrm{L} \\
\text { (centi- } \\
\text { mètres } \\
\text { cubes) }\end{array}$ & $A+1$ & FI. & AT $\%$ & AP \% & $1 \%$ \\
\hline 34 & $\ldots \ldots \ldots$ & 5 & 0 & - & 20,00 & 20,00 & 0,00 \\
\hline 35 & $\ldots \ldots$ & 4 & 1 & & 15,00 & 16,00 & $-1,00$ \\
\hline 36 & $\ldots \ldots$ & 3 & 2 & & 11,00 & 12,00 & $-1,00$ \\
\hline 37 & $\ldots \ldots$ & 2 & 3 & 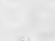 & 7,00 & 8,00 & $-1,00$ \\
\hline 38 & $\ldots \ldots$ & 1 & 4 & $i$ & 4,00 & 4,00 & 0,00 \\
\hline 39 & $\ldots \ldots \ldots$ & 0 & 5 & 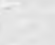 & 0,00 & 0,00 & 0,00 \\
\hline 40 & $\ldots \ldots$ & 5 & - & 0 & 18,00 & 18,00 & 0,00 \\
\hline 41 & $\ldots \ldots \ldots$ & 4 & - & 1 & 22,00 & 20,40 & 1,60 \\
\hline 42 & $\ldots$ & 3 & - & 2 & 25,00 & 22,80 & 2,20 \\
\hline 43 & $\ldots \ldots$ & 2 & - & 3 & 34,00 & 25,20 & 8,80 \\
\hline 44 & $\ldots \ldots$ & 1 & - & 4 & 24,00 & 27,60 & $-3,60$ \\
\hline 45 & $\ldots \ldots \ldots$ & 0 & - & 5 & 30,00 & 30,00 & 0,00 \\
\hline 46 & $\ldots \ldots$ & 5 & 0 & - & 20,00 & 20,00 & 0,00 \\
\hline 47. & & 4 & I & - & 15,00 & 16,00 & $-1,00$ \\
\hline 48 & $\ldots$ & 3 & 2 & $\longrightarrow$ & 10,00 & 12,00 & $-2,00$ \\
\hline 49 & $\ldots \ldots$ & 2 & 3 & - & 7,00 & 8,00 & 1,00 \\
\hline 50 & $\ldots$ & 1 & 4 & - & 5,00 & 4,00 & 1,00 \\
\hline 51 & $\ldots \ldots$ & 0 & 5 & - & 0,00 & 0,00 & 0,00 \\
\hline 52 & . & 5 & - & 0 & 18,00 & 18,00 & 0,00 \\
\hline 53 & $\therefore$ & 4 & - & 1 & 22,00 & 19,20 & 2,80 \\
\hline 54 & $\ldots \ldots$ & 3 & $\div$ & 2 & 22,00 & 20,40 & 1,60 \\
\hline 55 & $\ldots$ & 2 & $\div$ & 3 & 22,00 & 21,60 & 0,40 \\
\hline 56 & $\ldots$ & 1 & - & 4 & 24,00 & 22,80 & 1,20 \\
\hline 57 & & 0 & $\rightarrow$ & 5 & 24,00 & 24,00 & 0,00 \\
\hline
\end{tabular}

Signification des lettres majuscules :

$\mathrm{L}=$ lait $; \mathrm{Aq}=$ eau $; \mathrm{FL}=$ ferments lactiques ; $\mathrm{AT}=$ acidité totale ; $\mathrm{AP}=$ somme des acidités partielles; $\mathrm{I}=\mathrm{AT}-\mathrm{AP}=$ indice d'acidification.

décroissant, comme on devait s'y attendre. La présence de l'eau diminue l'acidogénie puisque les I sont négatifs, acidogénie d'autant moins prononcée qu'il y a plus d'eau, sauf pour le rapport $\frac{\mathrm{L}}{\mathrm{Aq}}=\frac{4}{\mathrm{I}}$. La dilution contrarie done la fermentation lactique. 
En présence de FL (tubes 17 à 21) la dilution du lait donne également une acidogénie progressivement décroissante, mais cette décroissance, beaucoup moins active, se traduit par une acidogénie positive, somme de l'acidité primitive apportée par FL au moment du mćlange et de l'acidité provoquée par sa présence, grâce à la fermentation lactique du lait ; donnant ainsi des I positifs avec un maximum pour $\frac{\mathrm{L}}{\mathrm{FL}}=\frac{3}{2}$.

TABLEAU II

DOSAGE dE L'AGIDITE APRÉs 24 HEURES DE SÉJOUR AU LABORATOIRE

(Température moyenne 15 à $\left.20^{\circ} \mathrm{C}.\right)$

\begin{tabular}{|c|c|c|c|c|c|c|c|}
\hline & Tubes & $\begin{array}{l}\text { (centi- } \\
\text { mètres } \\
\text { cubes) }\end{array}$ & $\mathbf{A}_{q}$ & FL & AT $\%$ & AP $\%$ & I \% \\
\hline 11 & $\ldots$ & 4 & 1 & - & 48,00 & 50,40 & $-2,40$ \\
\hline 12 & $\cdots$ & 3 & 2 & - & 37,00 & 37,80 & $\longrightarrow 0,80$ \\
\hline 13 & $\ldots$ & 2 & 3 & - & 22,00 & 25,20 & $-3,20$ \\
\hline 14 & $\ldots$ & 1 & 4 & - & 9,00 & 12,60 & $-3,60$ \\
\hline 15 & $\ldots$ & 0 & 5 & - & 0,00 & 0,00 & 0,00 \\
\hline 16 & $\ldots$ & 5 & 0 & - & 63,00 & 63,00 & 0,00 \\
\hline 17 & . & 4 & - & 1 & 58,00 & 55,60 & 2,40 \\
\hline 18 & $\ldots \ldots$ & 3 & - & 2 & 55,00 & 48,20 & 6,80 \\
\hline 19 & $\ldots \ldots$ & 2 & $\rightarrow$ & 3 & 42,00 & 40,80 & 1,20 \\
\hline 20 & & 1 & - & 4 & 36,00 & 33,40 & 2,60 \\
\hline 21 & & 0 & - & 5 & 26,00 & 26,00 & 0,00 \\
\hline
\end{tabular}

Avec FL (tableau I, tubes 40 à 45) celui-ci n'ayant pas le temps de provoquer une fermentation du lait, doit simplement ajouter son acidité à l'acidité primitive de L. On constate en effet que les AT de L + FL sont supérieurs à ceux de L + Aq; que, de plus, les I sont progressivement croissants tandis qu'ils étaient nuls avec $\mathrm{L}+\mathrm{Aq}$. Un seul point singulier concerne le rapport $\frac{\mathrm{L}}{\mathrm{FL}}=\frac{1}{4}$ (tube 44).

Si l'on refait les mêmes expériences de dosage immédiat avec les mélanges $\mathrm{L}+\mathrm{Aq}$ et $\mathrm{L}+\mathrm{FL}$ (tubes 46 à 57) on obtient des résultats sensiblement identiques. 


\section{Acidogénie après 24 heures de congélation variant de $-14^{\circ}$ à $-3^{\circ}$}

Dans le tableau III (tubes 28 à 33 et 22 à 27 ), les deux expériences de dosage immédiat donnent sensiblement les mêmes chiffres avec des I légèrement inférieurs à zéro, mais très voisins de zéro. Après congélation de 24 heures, les AT sont progressivement décroissants avec la dilution, comme il convient, mais restent inférieurs aux AT des dosages primitifs immédiats avant toute congélation. Le froid entraînerait-il donc une diminution de l'acidité à partir de ce moment et par quel mécanisme ? Comme dans le dosage immédiat, la dilution entraîne aussi une diminution de cette acidité, mais moins sensible, les I étant encore plus proches de zéro.

TABLEAU III

DOSAGE DE L'AGIDITÉ APRÉS GONGELATION DE 24 HEURES

VARIABLE DE $-14^{\circ} A-3^{\circ}$

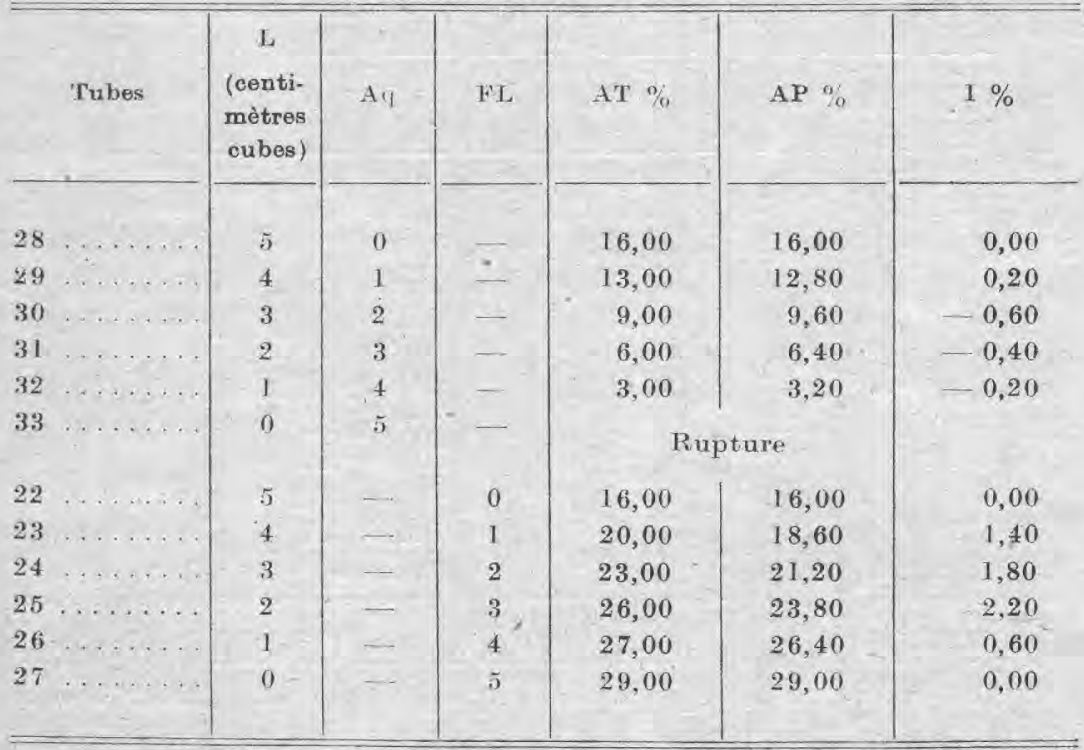

Dans les deux expériences du dosage immédiat on n'a pas exactement les mêmes nombres, FL étant d'une acidité de 30 d'un côté et de 24 de l'autre. Aussi faut-il comparer le tableau L + FL après congélation, qui est unique et qui donne 29 pour FL, au tableau de dosage immédiat qui donne 30 pour FL. On constate tout d'abord que pour $\mathrm{L}$ seul on a 16 après congélation au lieu de 18 , ce qui vient confirmer que le froid paraît abaisser le degré acidimétrique. Rien d'étonnant à ce que les autres nombres des mélanges restent inférieurs, sauf pour le mélange $\frac{\mathrm{L}}{\mathrm{FL}}=\frac{1}{4}$ où l'on trouve 27 
après congélation (tube 26) au lieu de l'acidité primitive 24 (tube 44), ce qui entraîne un I positif pour le premier et négatif pour le second, ici véritablement anormal. Naturellement les I sont supérieurs dans le dosage immédiat. Cela s'explique si l'on admet que l'acidité de FL ne change pas pendant la congélation, tandis que seule diminuerait celle de L.

\section{Deuxième série d'expériences}

Les dilutions aqueuses (tableau IV, tubes de 72 à 75 ) montrent à la réception une acidogénie progressivement décroissante, avec des I voisins de zéro. En réalité ils devraient être exactement nuls,

TABLEAU IV

DOSAGE DE L'AGIDITÉ A LA TEMPÉRATURE DU LABORATOIRE $\left(15-18^{\circ} \mathrm{G}\right)$

\begin{tabular}{|c|c|c|c|c|c|c|c|c|c|c|c|}
\hline \multirow{2}{*}{$\begin{array}{l}\text { Tu- } \\
\text { bes }\end{array}$} & \multirow{2}{*}{$\begin{array}{c}\mathrm{L} \\
\text { (eenti- } \\
\text { mètres } \\
\text { cubes) }\end{array}$} & \multirow{2}{*}{ Aq } & \multicolumn{3}{|c|}{ A la réception } & \multicolumn{3}{|c|}{$\begin{array}{c}\text { Après } 24 \text { heures de } \\
\text { laboratoire }\end{array}$} & \multicolumn{2}{|c|}{ Total des } & \multirow{2}{*}{ Total des I $+I^{\prime}$} \\
\hline & & & $\begin{array}{l}\mathrm{AT} \\
\%\end{array}$ & $\begin{array}{c}\mathrm{AP} \\
\%\end{array}$ & $1 \%$ & $\begin{array}{c}A^{\prime} T^{\prime} \\
\%\end{array}$ & $\left|\begin{array}{c}A^{\prime} \mathbf{P}^{\prime} \\
\%\end{array}\right|$ & I $\%$ & $\begin{array}{c}\mathrm{AT}+ \\
\mathrm{A}^{\prime} \mathrm{T}^{\prime}\end{array}$ & AP + & \\
\hline 72 & 5 & 0 & 16,40 & 16,40 & 0,00 & 34,00 & 34,00 & 0,00 & 50,40 & 50,40 & $0,00+0,00=0,00$ \\
\hline 73. & 3 & 2 & 9,00 & 9,80 & $-0,80$ & 19,00 & 20,40 & 1,40 & 28,00 & 30,20 & $-0,80+-1,40=-2,20$ \\
\hline 74. & 2 & 3 & 5,00 & 6,60 & $-1,60$ & 15,00 & 13,60 & 1,40 & 20,00 & 20,20 & $-1,60++1,40=-0,20$ \\
\hline 75 & 0 & 5 & 0,00 & 0,00 & 0,00 & 0,00 & 0,00 & 0,00 & 0,00 & 0,00 & $0,00+-0,00=-0,00$ \\
\hline 78 & 5 & 0 & - & - & $一$ & 78,00 & 78,00 & 0,00 & - & - & 一 \\
\hline 79 & 3 & 2 & - & $=$ & - & 49,00 & 46,80 & 2,20 & - & $\longrightarrow$ & - \\
\hline 80 & 2 & 3 & - & 一 & - & 35,00 & 31,20 & 3,80 & - & - & - \\
\hline 81 & 0 & 5 & - & $=$ & - & 0,00 & 0,00 & 0,00 & - & - & \\
\hline 83 & 5 & 0 & 一 & - & 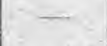 & 78,00 & 78,00 & 0,00 & - & - & - \\
\hline
\end{tabular}

puisque l'eau est neutre à la phtaléine. Les légers écarts qu'ils présentent avec le zéro peuvent s'expliquer par une erreur d'une goutte en plus ou en moins de la liqueur alcaline, cette goutte étant égale au $1 / 20^{\mathrm{e}}$ de centimètre cube.

Les acidités prises sur les mêmes tubes après 24 heures de séjour au laboratoire, ces tubes étant déjà neutralisés par la soude à la réception, montrent un travail d'acidogénie que nous pouvons comparer avec celui qui a été provoqué après 24 heures depuis la réception, sans neutralisation au moment de cette réception.

On remarque que les totaux des acidogénies AT + A'T' demeurent inférieurs aux acidogénies sans neutralisation préalable (tableau IV, tubes de 78 à 83). Done, dans le cas particulier envisagé, les fermentations acidogènes, au lieu d'être accélérées par la neutra- 
lisation, comme il arrive souvent, ont été au contraire, ici, freinées. De plus, les I qui demeurent presque nuls dans le premier cas, sont augmentés dans le second, la dilution entraînant une accélération de l'acidogénie.

Après congélation, on remarque (tableau $V$, tubes 90 à 94 ) que les mélanges $\mathrm{L}+\mathrm{Aq}$ fournissent des AT sensiblement égaux à ceux de la réception, les I ayant aussi sensiblement la même valeur voisine de zéro. Par conséquent, la congélation n'a pas influencé l'acidogénie.

Par suite d'un accident, les résultats numériques relatifs aux mélanges L + FL n'ont pu être recherchés, mais la première série d'expériences et les autres antérieurement publiées ont démontré nettement l'action négative de FL pendant la congélation.

TABLEAU $V$

DOSAGE DE L'AGIDITÉ APRÈS GONGÉLATION DE 24 HEURES VARIABLE DE $-15^{\circ} A-5^{\circ}$

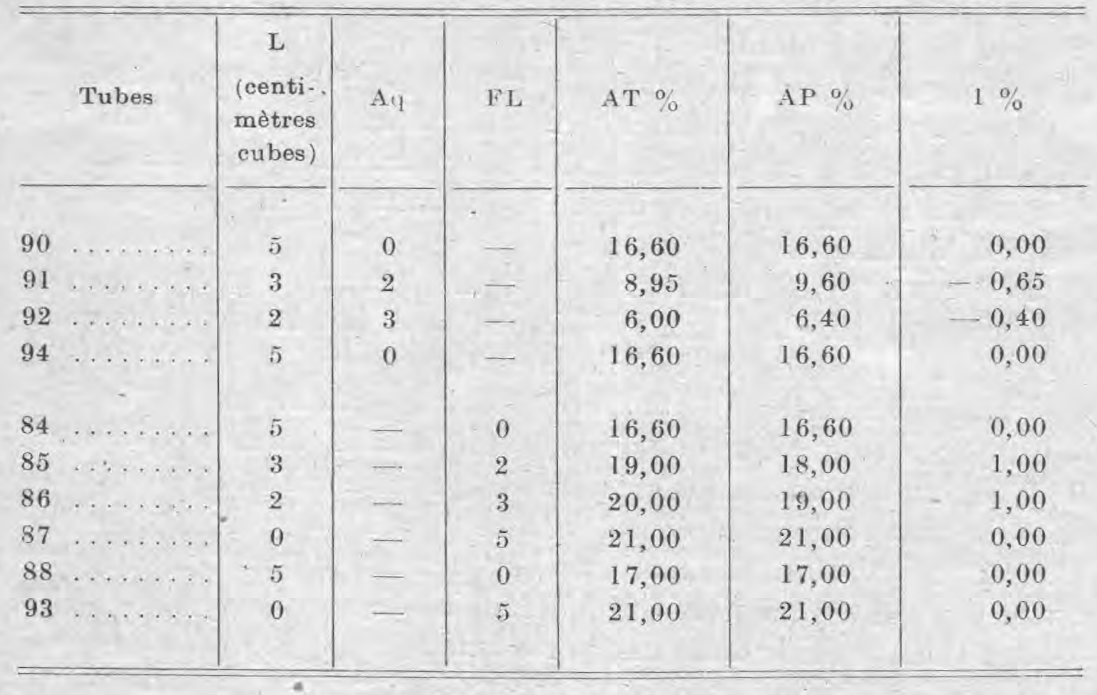

\section{Conclusions}

L'acidogénie du lait, soit spontanée, soit par son mélange avec FL, après congélation de 24 heures, semble nulle. En effet, les Indices d'Acidification I avoisinent zéro avec un écart négligeable et sont sensiblement égaux à ceux qui proviennent des dosages primitifs immédiatement avant toute congélation.

Par contre, après 24 heures, sans congélation, l'acidogénie se manifeste aussi bien avec dilution par le mélange du lait avec l'eau, qu'avec dilution par son mélange avec la culture FL de ferments lactiques. 
Et, tandis que, dans le premier cas, le lait fermente seul spontanément, dans le second cas, l'intervention fermentative de FL ajoutée se manifeste en s'additionnant à la précédente.

Ainsi, e'est bien la eongélation qui supprime toute fermentation acidogène.

D'autre part, d'autres expériences ont prouvé l'inaltérabilité du bleu de méthylène pendant la congélation et par conséquent l'inaction des réductases. Les autres diastases, d'après cela, demeurent aussi inactives et par conséquent sans action sur la composition du lait.

De plus le $\Delta$ du lait ne changé pas, après coñgélation, non plus que son aspect et sa saveur, qu'il soit examiné aussitôt après décongélation ou après ébullition (1).

A partir de ce moment se comporte-t-il comme le lait non congelé ou bien se conserve-t-il ou s'altère-t-il davantage ?

S'il existe des modifications dans sa composition, elles doivent porter de préférence sur les protides. Elles semblent peu probables d'après ce qui précède. Des analyses comparatives et des expériences physiologiques seraient à reprendre, car les conclusions des auteurs rappelées au début de cet article ne paraissent pas convaincantes, après lecture attentive de leurs communications ne comportant d'ailleurs pas de données numériques ni de détails expérimentaux.

En outre, je mentionne qu'un examen microscopique comparatif ne révèle aucune différence entre le lait décongelé et le même lait examiné à sa réception.

Dans des recherches poursuivies du 23 janvier au 1 er février 1940, j'ai obtenu des conclusions semblables aux précédentes. Mais, ici, au lieu de maintenir les mélanges sous un froid graduellement et très lentement décroissant de $-15^{\circ}$ à $-5^{\circ}$, j'ai voulu profiter des variations nocturnes et diurnes de la température extérieure qui a varié très irrégulièrement de $-10^{\circ}$ à $+10^{\circ}$.

Les tubes, réunis dans des porte-tubes grillagés, étaient placés circonférenciellement contre le grillage pour être exposés aussi également que possible aux variations de température. Le portetubes, exposé au sixième et dernier étage d'une maison et placé sur un support, était lui-même protégé contre les offenses des troubles atmosphériques : neige, pluie, vent et radiations lumineuses, par un chapeau métallique. Seules les variations thermiques pouvaient atteindre les mélanges étudiés. Des tubes contenant les mêmes mélanges restèrent à l'intérieur du laboratoire à une température ayant varié de 15 à $17^{\circ} \mathrm{C}$.

(1) Les mêmes expériences comparatives ont été exécutées dans le même temps avec des œufs frais et les mêmes résultats ont été constatés. 
Tant que le lait est resté congelé, qu'il fût dilué ou non soit par Aq, soit par FL, son acidogénie put s'identifier à l'acidogénie du même lait à sa réception au laboratoire: J'ai pu également faire des comparaisons entre les acidogénies dès la décongélation des mélanges et les acidogénies des mêmes mélanges maintenus au laboratoire pendant la même durée, à une température moyenne de $15^{\circ}$. Je n'en donnerai pas les tableaux numériques puisqu'il s'agit ici de ceux qui eoneernent seulement la congélation.

Voici maintenant les résultats de mes recherches portant sur une durée beaucoup plus étendue puisqu'elles se sont exercées pendant plus d'un mois, mais seulement à une température de -5 $\grave{a}-6^{\circ}$.

J'ai examiné les propriétés organoleptiques du lait, son $\Delta$, l'état de ses réductases et son aspect microscopique. J'ai comparé toutes ces données aux données correspondantes du même lait, à sa réception, et aussi à différentes durées et à différentes températures : celle du laboratoire et à des températures où l'action microbienne est plus ou moins annihilée par la chaleur, jusqu'à suppression complète de cette action microbienne. J'ai opéré dans les mêmes conditions qu'antérieurement, à savoir sur un volume aussi réduit que possible eapable d'épouser rapidement les variations de température. Pour bień mettre en évidence l'action microbienne d'acidogénie lactique, j'ai dilué le lait sous volume constant avec une culture très active de ferments lactiques. Pour tenir compte de cette dilution, elle a été déjà examinée, pour les mêmes mélanges à volume constant du lait, avec de l'eau pure, dans notre précédent travail.

Je rappelle ici les notations déjà adoptées dans mes recherches antérieures :

$$
\mathrm{v}+\mathrm{v}^{\prime}=5 \mathrm{cc} .
$$

où $v$ représente le volume de $L$ variable de 5 à 0 , inversement $v^{\prime}$ le volume de $\mathrm{FL}$ ou de Aq, L désignant le lait, FL la culture de ferments lactiques, Aq l'eau pure :

$$
\mathrm{v}^{\mathrm{p}}+\mathrm{v}^{\prime} \mathrm{p}^{\prime}=\mathrm{AP}
$$

où $\mathrm{p}$ et $\mathrm{p}^{\prime}$ sont les acidités partielles calculées respectivement de $v$ et $v^{\prime}$, AP représentant l'acidité totale calculée du mélange.

Si l'on désigne par AT l'acidité totale fournie par l'expérience, on aura un Indice d'acidification:

$$
\mathrm{I}=\mathrm{AT}-\mathrm{AP}
$$

Les résultats numériques rassemblés dans les tableaux ci-joints ont été fournis par neutralisation avec $\mathrm{NaOH}, \mathrm{N} / 10$ en présence de Phtaléine. 
DOSAGE des AGIDITÉS AU COURS de LA GONGELATION

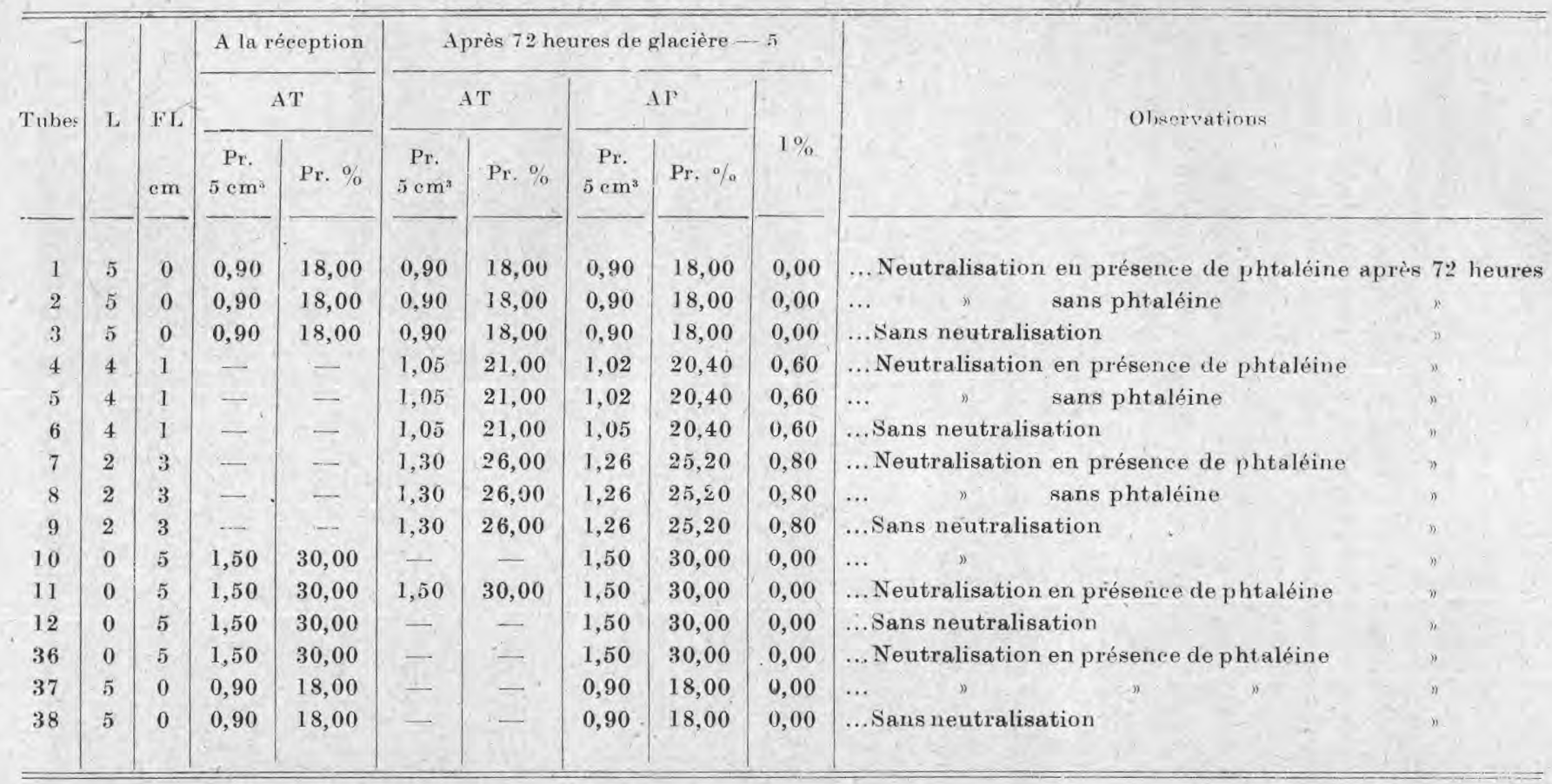

Observations. - Après 72 heures de glacière à - $5^{\circ}$ on constate que le contenu de tous les tubes est congelé. La congélation est d'autant moins rapide que les tubes sont plus riches en FL. La culture pure est la plus rebelle.

Après plus d'un mois de glacière, l'acidogénie est encore demeurée nulle, aussi bien dans les tubes sans neutralisation que dans les tubes neutralisés en présence d'une goutte de phtaléine à $1 \%$ ou sans la présence de phtaléine, les nombres représentant la neutralisation de ces derniers étant déterminés par ceux des tubes qu'on a neutralisés en présence de phtaléine. Ceci afin de savoir si une goutte de phtaléine a une influence sur l'acidogénie. L'action de FL demeure done nulle pendant la congélation. 
TEMPÉRATURE ORDINAIRE DU LABORATOIRE (15-18०)

DOSAGE DES ACIDITÉS APRÈS GONGÉLATION ET DU MÊME LAIT NON CONGELÉ A LA TEMPÉRATURE ORDINAIRE

DU LABORATOIRE $\left(1^{\left.5-18^{\circ}\right)}\right.$ PENDANT LES MÊMES DURÉES

\begin{tabular}{|c|c|c|c|c|c|c|c|c|c|c|c|c|c|}
\hline \multirow{3}{*}{ Tubes } & \multirow{3}{*}{ L } & \multirow{3}{*}{ FL } & $\begin{array}{l}\text { A la ré- } \\
\text { ception }\end{array}$ & \multicolumn{5}{|c|}{ Après 24 heures } & \multicolumn{4}{|c|}{ Après 48 heures } & 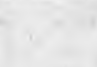 \\
\hline & & & $\mathrm{AT}$ & \multicolumn{2}{|c|}{$\mathrm{AT}$} & \multicolumn{2}{|c|}{$A P$} & \multirow{2}{*}{$\mathrm{I} \%$} & \multicolumn{2}{|c|}{$\mathrm{AT}$} & \multicolumn{2}{|c|}{$A P$} & \multirow{2}{*}{$1 \%$} \\
\hline & & & Pr. $5 \mathrm{~cm}^{3}$ & Pr. $5 \mathrm{~cm}^{3}$ & Pr. \% & Pr. $5 \mathrm{~cm}^{3}$ & P. $\%$ & & Pr. $5 \mathrm{~cm}^{3}$ & Pr. \% & Pr. $5 \mathrm{~cm}^{3}$ & Pr. $\%$ & \\
\hline 13 & 5 & 0 & 0,90 & 1,30 & 26,00 & 1,30 & 26,00 & 0,00 & 4,70 & 94,00 & 4,70 & 94,00 & 0,00 \\
\hline 14 & 5 & 0 & 0,90 & 1,50 & 30,00 & 1,50 & 30,00 & 0,00 & 5,60 & 112,00 & 5,60 & 112,00 & 0,00 \\
\hline 15 & 5 & 0 & 0,90 & - & - & - & - & $\rightarrow$ & 4,30 & 86,00 & 4,30 & 86,00 & 0,00 \\
\hline 16 & 4 & 1 & - & 1,40 & 28,00 & 1,02 & 20,40 & 7,60 & 5,10 & 102,00 & 1,02 & 20,40 & 81,60 \\
\hline 17 & 4 & 1 & - & 1,40 & 28,00 & 1,02 & 20,40 & 7,60 & 5,30 & 106,00 & 1,02 & 20,40 & 85,60 \\
\hline 18 & 4 & 1 & - & - & - & $\sim$ & - & - & 3,70 & 74,00 & 1,02 & 20,40 & 53,60 \\
\hline 19 & 2 & 3 & - & 1,40 & 28,00 & 1,26 & 25,20 & 2,80 & 1,80 & 36,00 & 1,26 & 25,60 & 10,40 \\
\hline 20 & 2 & 3 & $\rightarrow$ & 1,40 & 28,00 & 1,26 & 25,20 & 2,80 & 2,40 & 48,00 & 1,26 & 25,60 & 22,40 \\
\hline 21 & 2 & 3 & $=$ & - & - & $\longrightarrow$ & $\longrightarrow$ & $\ldots$ & 2,00 & 40,00 & 1,26 & 25,60 & 14,40 \\
\hline 22 & 0 & 5 & 1,50 & 1,50 & 30,00 & 1,50 & 30,00 & 0,00 & 1,40 & 28,00 & 1,40 & 28,00 & 0,00 \\
\hline 23 & 0 & 5 & - & $\div$ & - & - & - & $\rightarrow$ & 1,40 & 28,00 & 1,40 & 28,00 & 0,00 \\
\hline 24 & 0 & 5 & 1,50 & 1,60 & 32,00 & 1,60 & 32,00 & 0,00 & 1,60 & 32,00 & 1,60 & 32,00 & 0,00 \\
\hline
\end{tabular}

Observations. - Si l'on compare après congélation les acidogénies du lait, à la température ordinaire, à l'acidogénie du lait à la même température ordinaire mais n'ayant pas subi de congélation, on obtient des résultats numériques sensiblement identiques. Nous ne reproduisons donc qu'un seul des deux tableaux représentatifs des acidogénies du lait congelé et non congelé.

Les dosages d'acidogénie ont été poursuivis même après coagulation des mélanges, tous coagulés au bout de 48 heures. Le parallélisme d'identification acidogène existe toujours entre les laits congelé et non congelé.

On constate que la marche des acidogénies n'est pas non plus modifiée entre les deux laits dans les tubes oì nous avons, entre temps, neutralisé leur contenu en présence ou non de phtaléine. 


\section{Conclusions}

Les conclusions relatives à la congélation du lait ne laissent point de doute quant à son acidogénie qui demeure nulle après plus d'un mois de eongélation, quel que soit le moment du dosage de cette acidogénie. Aueune basogénie non plus n'est constatée, dans les mêmes conditions. Cela ne suffit évidemment pas à prouver que le lait a conservé son équilibre bio-physico-chimique, mais d'autre part ses réductases, appréciées au bleu de méthylène, n'entrent en activité qu'après relèvement de la température. Son $\Delta$ est constant. Quand on réchauffe ce lait progressivement, on observe exactement les mêmes phénomènes qu'avec le lait à la réception, malgré la période de temps (d'ailleurs assez courte, en raison des faibles volumes expérimentés) qui s'écoule jusqu'au moment où les mélanges congelés ont atteint la température ambiante. A cette température de $18^{\circ}$ environ, le lait dépourvu de eulture artificielle de ferments lactiques, ne se coagule qu'après le même temps que le lait non congelé, c'est-à-dire plus de 40 heures; par contre, décoloration très active du bleu de méthylène. - A $37^{\circ}$, coagulation après 15 heures environ de séjour à l'étuve et décoloration du bleu après environ une heure. - A $60^{\circ}$ pendant $30^{\prime}$ pas de coagulation deux jours après à la température ambiante, décoloration beaucoup plus tardive ou même supprimée quand les températures dépassent $70^{\circ}$. A toutes ees températures, jusqu'à $120^{\circ}$, on peut observer des propriétés identiques pour les deux laits, notamment au point de vue de leur acidité, de leur aspect, de leur goût et de leurs réductases, ces dernières présentant un maximum d'activité à la température ambiante, activité déclinant à $37^{\circ}$ et annihilée définitivement à partir de $70^{\circ}$ comme elle l'est temporairement pendant la congélation.

Pousser plus avant les recherches pour se faire une conviction paraît done inutile. Cependant, nous espérons apporter d'autres preuves et par des analyses chimiques comparatives complètes et par la recherche des hormones qui, sans nul doute, existent dans le lait, aliment de la première enfance. On retrouve, comme on le sait, ces hormones avec toutes leurs propriétés dans les glandes à sécrétion interne qui ont subi une congélation même de longue durée. Il est vrai que parfois l'architecture glandulaire et tissulaire des substances animales se trouve modifiée par le froid, mais leurs propriétés biologiques n'en paraissent pas sensiblement modifiées.

BIBLIOGRAPHIE

A consulter dans :

Bulletin de la Société de Chimie biologique, février 1938, p. 198.

Le Lait, t. XIX, no 186, juin 1939, p. 584-585. 